\title{
Liver transplantation for colo-rectal liver metastasis: survival without recurrence can be achieved
}

(2)

Christian Toso ${ }^{1}$, Hugo Pinto Marques ${ }^{2}$, Axel Andres ${ }^{1}$, Francisco Castro Sousa ${ }^{3}$, René Adam $^{4}$, Antonio Kali1 ${ }^{5}$, Pierre-Alain Clavien ${ }^{6}$, Emanuel Furtado ${ }^{7}$, Eduardo Barroso ${ }^{2}$, Henri Bismuth", on behalf of the "Compagnons hépato-biliaires" group

${ }^{1}$ Divisions of transplant and abdominal surgery, and Hepato-bilio-pancreatic centre, University of Geneva Hospitals, Geneva, Switzerland, ${ }^{2}$ Hepato-Biliary-Pancreatic and Transplantation Centre, Curry Cabral Hospital, Lisbon, Portugal, ${ }^{3}$ Department of Surgery A, University Hospital of Coimbra, Coimbra, Portugal, ${ }^{4}$ Centre Hépato-Biliaire, AP-HP Hôpital Paul Brousse, Villejuif, France, ${ }^{5}$ Department of Surgical Oncology, Hospital Santa Rita, do Complexo Hospitalar da Irmandade Santa Casa de Misericórdia de Porto Alegre, Porto Alegre, Brazil, ${ }^{6}$ Department of Surgery and Transplantation, University Hospital Zurich, Swiss HPB and Transplant Center, Zurich, Switzerland, ${ }^{7}$ Department of Surgery A, and Children and Adult Liver Transplantation Unit, University Hospitals of Coimbra, Coimbra, Portugal

Key words: recurrence, disease-free, complication, morbidity, chemotherapy

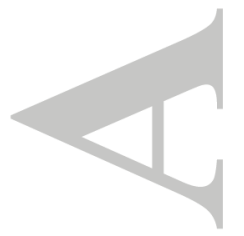

This article has been accepted for publication and undergone full peer review but has not been through the copyediting, typesetting, pagination and proofreading process which may lead to differences between this version and the Version of Record. Please cite this article as an 'Accepted Article', doi: 10.1002/lt.24791 


\section{Footnote page}

Alphabetical list of all abbreviations: ALPPS: associating liver partition and portal vein ligation for staged hepatectomy, CEA: carcinoembryonic antigen, mTOR: mammalian Target-of-Rapamycin, RFA: radio-frequency ablation,

Grants and financial support: Christian Toso was supported by the Swiss National Science Foundation (PP00P3_139021).

Conflict of interest: none

Correspondence: Christian Toso, Abdominal and Transplant Surgery, Geneva University Hospitals, Rue Gabrielle-Perret-Gentil, 1211 Geneva, Tel: +41223727639, Fax:

+41223727766, E-mail: christian.toso@hcuge.ch

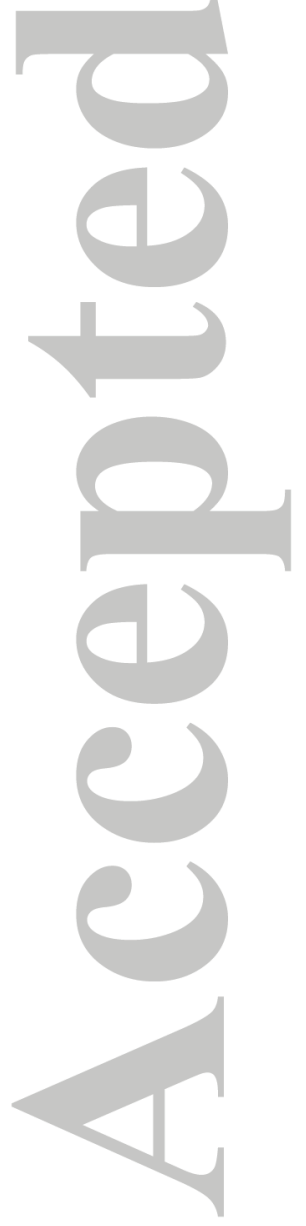

John Wiley \& Sons, Inc. 


\section{To the Editor,}

The management of patients with colo-rectal liver metastases has improved significantly over the recent years. However, most patients still cannot undergo complete resection, generally because the location of the metastases within the liver prevents any radical management, and explaining the interest for transplantation. The first attempts were performed in the eighties with poor outcomes, in part due to a high proportion of death not related to the neoplastic disease (1). More recently, the group of Oslo has shown a $60 \%$ five-year survival, in 21 patients with colo-rectal metastases (2). Such a survival was better than the one expected on chemotherapy alone (2-4). However, 95\% (20/21) of patients had cancer recurrence, most within the first 18 months after transplantation. At present, liver transplantation for colo-rectal metastases remains highly controversial. The potential for long-term disease-free survival needs to be explored, which is the aim of this multi-centric collaborative retrospective study.

A total of 12 patients ( 6 females/ 6 males) underwent liver transplantation for colo-rectal liver metastasis, at centers affiliated to the "Compagnons Hépato-Bilaires", an association of hepato-pancreato-biliary and transplant surgeons, most trained at the Paul Brousse Hospital, Paris, France under the guidance of Professor H. Bismuth. Median age at transplant was 56 years (Table 1). Patients were managed in Lisbon (n=8), Coimbra $(n=2)$, Paris $(n=1)$, and Geneva $(n=1)$ between October 1995 and October 2015 (date of transplant), and no other patient underwent transplantation for this indication at these respective transplant centers. Data collection was conducted according the relevant ethical standards at each institution. The location of the primary adenocarcinoma was the colon in 11 patients, and the rectum in one. Most primary cancers were T3 on pathology, 
and many presented between 1 and 3 involved -N1- lymph nodes (two patients were N2 with more than 3 nodes involved, Table 1).

For most patients, liver metastases $(9 / 12)$ were diagnosed within 12 months after the diagnosis of the primary cancer, and were considered as synchronous. When not diagnosed at the same time as the primary, liver metastases were discovered 4, 7, 19, 24 and 29 months after the primary. At the time of transplantation, patients presented a median of 9 liver metastases. Two had lesions $>5 \mathrm{~cm}$, of 5.5 and $8 \mathrm{~cm}$. Median CEA level was $16.9 \mu \mathrm{g} / 1$, and one patients had CEA $>200 \mu \mathrm{g} / 1$, of $314 \mu \mathrm{g} / 1$.

Most (11/12) patients received chemotherapy prior to transplantation. Chemotherapy included irinotecan and oxaliplatin in $9(82 \%)$ patients, and a biological agent in 6

(cetuximab in 2, bevacizumab in 3, and both agents in 1). Another patient was treated by intra-hepatic chemotherapy prior to transplantation. All patients responded to chemotherapy, and none was in progression at the time of the transplantation. The decision to conduct a post-transplant adjuvant chemotherapy was based, at least in the recent years, on the aim to obtain a minimum of four months of peri-transplant oxaliplatine and/or irinotecan-including chemotherapy. It was used in four patients.

Overall, most patients underwent complex, long-term chemotherapy treatment, with a median of two lines ( 1 in 1 patient, 2 in 6 patients, 3 in 2 patients, and 4 in 1 patient). In addition to chemotherapy, 10 patients underwent liver metastasis resection, in one $(n=5)$, two $(n=3)$, or three $(n=2)$ procedures, one of which was a two-stage resection. One patient underwent radio-frequency ablation (RFA) as part of the treatment. At the time of transplantation, any treatment by resection or RFA was not possible anymore. 
Transplantations were performed 41 (12-97) months after the resection of the primary. Liver grafts were from 6 non-marginal donors after brain-death, 5 domino donors with familial amyloid polyneuropathy, and one living-related donor. Transplantation was part of a planned treatment strategy following previous liver resection in 6 patients. In 2 patients, transplantation was performed upfront without previous resection, considered impossible because of diffuse liver disease. In three patients, transplantations were performed as salvage procedures: after massive bleeding (and packing) following liver resection, for a failed associating liver partition and portal vein ligation for staged hepatectomy (ALPPS) procedure, and for post-resection liver failure. Finally, one patient was transplanted with inferior vena cava replacement due to tumor invasion. Overall, 6 patients could be categorized as having planned transplantations, and 6 patients could be retrospectively considered as having received "compassioned" transplantations.

The median length of hospital stay for transplantation was 16 days. Seven patients had complications, including 3 patients with grade 2 complications according to ClavienDindo (pneumonia and fever of unknown origin treated by antibiotics, and rejection treated by steroids), 1 patients with grade $3 \mathrm{~b}$ complication (biliary leak requiring endoscopic stenting), 2 patients with grade 4 a complication (kidney failure requiring dialysis, prolonged ventilation requiring a tracheostomy), and 1 patient with grade 5 complication (death following severe bleeding and shock). Eight patients received mTOR inhibitors as part of their maintenance immunosuppression.

Overall patient survivals were $83 \pm 11 \%, 62 \pm 15 \%$, and $50 \pm 16 \%$ at 1,3 and 5 years, after a median follow-up of $26(0-108)$ months (Figure 1). Six patients had a recurrence, affecting the lungs $(n=5)$, the liver $(n=3)$, and the peritoneum $(n=1)$. Disease-free 
survivals were $56 \pm 14 \%, 38 \pm 15 \%$, and $38 \pm 15 \%$ at 1,3 and 5 years. Treatment of recurrence included palliative chemotherapy in five patients and radiotherapy in one. Five patients were alive and free of cancer $7,43,47,48$, and 108 months after transplantation (Table 1).

Patients undergoing transplantation for compassionate indications (upfront, salvage, and vena cava involvement) had significantly worse disease-free survivals than patients with planned indications $(\mathrm{p}=0.02)$. Looking at the Oslo criteria (2), the last pre-transplant CEA level ( $\geq 80$ vs. $<80 \mu \mathrm{g} / 1, \mathrm{p}=0.09)$ and the time between resection of the primary cancer and transplantation ( $\geq 24$ vs. $<24$ months, $\mathrm{p}=0.01$ ) also tended to predict disease-free survival. The size of the largest metastasis at transplant $(\geq 5.5 \mathrm{vs} .<5.5 \mathrm{~cm})$ failed to predict survival $(p=0.47)$.

As a whole, the present investigation goes one step further compared to the Norwegian experience, as it demonstrates that disease-free survival can be achieved. However, such an outcome was only seen in patients after transplantation performed as deliberate procedures in a long-term treatment $(n=6)$. These patients had previously undergone a combined oncological/surgical management with multiple courses of chemotherapy, and often multiple liver resections. None had a progressive disease at the time of transplantation. Conversely, the six patients with upfront, emergency, and extrahepatic disease transplantations ("compassionate" indications) did significantly worse, and most had a recurrence within 18 months, suggesting that transplantation in these situations must be avoided.

In addition, the present cohort shows that the time between colo-rectal surgery and transplantation (with a cut-off at 24 months) and the last CEA level $(80 \mu \mathrm{g} / 1)$ tend to 
predict disease-free survival, while tumor diameter $(<5.5 \mathrm{~cm})$ and cancer progression (no patient in progression) could not be identified. These factors as a whole should be used and refined for selecting patients for prospective trials (short time between primary and transplant, low CEA, small lesion, without progression)(2).

Of note, the Norwegian experience (21 published patients) has been possible thanks to the unique high organ donor availability in this country, where the recipient needs are covered within a short waiting time. In all other countries, the lack of donors represent a major challenge to transplantation for colo-rectal liver metastasis. Outcomes still need to be improved and validated before potentially justifying a systematic use of liver grafts for this indication on a common waiting list with patients with end-stage liver failure and hepatocellular carcinoma. In the meantime, domino, and living-donor liver grafts should be favored, and the RAPID concept further explored (5). In addition, current transplantation for non-resectable colo-rectal metastasis should be conducted within prospective trials with strict inclusion and exclusion criteria.

The present study should be seen as a proof-of-feasibility, demonstrating the potential of achieving disease-free survival after transplantation colo-rectal metastases in a meaningful proportion of patients. It should encourage turning away from more emotional indications, in favor of prospective studies with strict selection criteria to explore the potential of this modality.

\section{Acknowledgements}


The authors thank Pietro Majno, for his help in revising the manuscript and in the drafting of the study protocol. They are also thankful to Sofia Corado for collecting patient data from Lisbon.

\section{“Compagnons Hépato-bilio-pancréatiques" group}

The "compagnons hépato-pancréato-biliaires" include hepato-pancreato-biliary and transplant surgeons, most trained in the Paul Brousse hospital. The following experts have been contacted in view of a workshop in Porto Alegre (April 2016), and have contributed to the work:

Rene Adam (Hôpital Paul Brousse, Villejuif, France), Dimitri Alden (New York, USA), Malcolm Aldridge (East and North Hertfordshire NHS, Hertford, UK), Oscar Andriani (Hospital Universitario Austral, Buenos Aires, Argentina), Arie Ariche (West Galile Medical Center, Nahariyya, Israel), Ibrahim Astarcioglu (Genel Cerrahi Anabilim Dali, Izmir, Turkey), Eduardo Barroso (Hospital de Curry Cabral, Lisbon, Portugal), Abdelkader Belkouchi, Hopital Avicenne, Rabbat, Maroc), Henri Bismuth (Hôpital Paul Brousse, Villejuif, France), Fernando Buabse (San Miguel de Tucuman, Argentina), Fulvio Calise (Ospedale Cardarelli, Napoli, Italy), Enrique Miguel Canepa (Hospital Privado de Comunidad, Cordoba, Argentina), Francisco Castro Sousa (Coimbra University Hospital, Coimbra, Portugal), Daniel Cherqui (Hopital Paul-Brousse, Villejuif, France), Laurence Chiche (Bordeaux University Hospital, Bordeaux, France), Miguel Ciardullo (Hospital Italiano, Buenos Aires, Argentina), Pierre-Alain Clavien (Zurich University Hospital, Zurich, Switzerland), Marvin Corlette (Belmont, CA, USA), Guido Costamagna (Universita Cattolica de Sacre Cuore, Rome, Italy), Joao Coutinho 
(Hospital St Maria, Lisbon, Portugal), Ignazia Demma (Anthea Hospital, Bari, Italy), Tom Diamond (Queen's University, Belfast, UK), Jiahong Dong (Chinese PLA General Hospital Chinese Beijing, China), Jean Edmond (Columbia University, New York, USA), Rachad El-Masry (Ahmed Maher Teaching Hospital , Cairo , Egypt), Rony Eshkenazy (Sheba Medical Center, Tel-Aviv, Israel), Olivier Farges (Hopital Beaujon, Clichy, France), Cyrille Feray (Hôpital Henri-Mondor, Paris, France), Dominique Franco (Hopital Antoine-Béclère, Clamart, France), Francisco Navarro Freire (University of Granada, Granada, Spain), O James Garden (University of Edinburgh, Edinburgh, UK), Jean-François Gigot (Cliniques Universitaires Saint Luc, Bruxelles, Belgium), Jean Gugenheim (Hopital de l'Archet II, Nice, France), Nagy Habib (Hammersmith Hospital,

London, UK), Ellen Hagopian (Jersey Shore University Medical Center, New Jersey, USA), Emir Hoti (St Vincent's University Hospital, Dublin, Ireland), John Isaac (Queen Elizabeth Medical Centre, Birmingham, UK), Marc Johann (Hopitaux Privés de Metz, Metz, France), Ousmane Ka (Université Cheikh Anta Diop, Dakar, Senegal), Antonio Kalil (Hospital Moinhos de Vento, Porto Alegre, Brazil), Andreas Katz (Greenville Medical Tower, Texas, USA), Jina Krissat (Blackpool Victoria Hospital, Blackpool, UK), Réal Lapointe (Centre Hospitalier de l’Université de Montréal, Montréal, Canada), Jan Lerut (Cliniques Universitaires Saint Luc, Bruxelles, Belgium), Cristiano Xavier Lima (Hospital Felice Rocho, Belo Horizonte, Brazil), Marcelo Linhares (Universidade Federal de Sao Paulo, Sao Paulo, Brazil), Valerio Lucidi (Erasme University Hospital, Bruxells Belgium), Pietro Majno (Geneva University Hospital, Geneva, Switzerland), Stelios Mantonakis (Errikos Dynan Hospital, Athens, Greece), Surendra Kumar Mathur (Medical College \& BYL Nair Hospital, Mumbai, India), Francesco Meriggi (University 
of Pavia, Pavia, Italy), Mario Morino (Istituto di Clinica e Terapia Chirurgica, Torino,

Italy), Richard Nakache (Tel-Aviv Souraski Medical Center, Tel-Aviv, Israel), Benoit

Navez (Cliniques Universitaires Saint-Luc, Bruxelles, Belgium), Gennaro Nuzzo

(Hepatobiliary Unit Largo R, Roma, Italy), Roger Obregon (Federal University of Sao

Paulo, Sao Paulo, Brazil), Raul Oleas (Quito, Ecuador), Jorge Paulino (Hospital de Curry

Cabral, Lisbon, Portugal), Riccardo Pellicci (Ospedale Santa Corona, Pietra Ligure,

Italy), Hugo Pinto Marques (Hospital de Curry Cabral, Lisbon, Portugal), Joseph Raccuia

(New York University Langone Medical Center, New York, USA), Juan Manuel Rico

Juri (Imbanaco Medical Center, Cali, Colombia), Antonio Robecchi (Turin, Italy),

Rodolfo Rossi (Sanatoria Mater Dei, Buenos Aires, Argentina), Daradkeh Salam (Jordan

University, Amman, Jordan), Faouzi Saliba (Hôpital Paul Brousse, Villejuif, France),

Didier Samuel (Hopital Paul Brousse, Villejuif, France), Carlos Eduardo Santos

(National Cancer Institute of Brazil, Botafogo, Brazil), Sabine Sarnacki-Feray (Hopital

Necker, Paris, France), Tzyy-Yuan Shann (Auckland, New Zealand), David Sherlock

(North Manchester General Hospital, Manchester, UK), Daniel Shouval (Hadassah

Hebrew University Hospital, Jerusalem, Israel), Christian Toso (Geneva University

Hospital, Geneva, Switzerland), Oscar Traynor (National Surgical Training Centre,

Dublin, Ireland), Françoise Van Hove (Hôpital Paul Brousse, Villejuif, France), Corinne

Vons (Hopital Jean-Verdier, Paris, France), Raja Wakim (Mount Lebanon Hospital,

Hazmieh, Lebanon), Krzysztof Zieniewicz (Warszawski Uniwersytet Medyczny,

Warsaw, Poland)

John Wiley \& Sons, Inc.

This article is protected by copyright. All rights reserved. 


\section{References}

1. Muhlbacher F, Huk I, Steininger R, Gnant M, Gotzinger P, Wamser P, et al. Is orthotopic liver transplantation a feasible treatment for secondary cancer of the liver? Transplant Proc. 1991;23(1 Pt 2):1567-8.

2. Hagness M, Foss A, Line PD, Scholz T, Jorgensen PF, Fosby B, et al. Liver transplantation for nonresectable liver metastases from colorectal cancer. Annals of surgery. 2013;257(5):800-6. 3. Dueland S, Hagness M, Line PD, Guren TK, Tveit KM, Foss A. Is Liver Transplantation an

Option in Colorectal Cancer Patients with Nonresectable Liver Metastases and Progression on All Lines of Standard Chemotherapy? Annals of surgical oncology. 2015;22(7):2195-200.

4. Dueland S, Guren TK, Hagness M, Glimelius B, Line PD, Pfeiffer P, et al. Chemotherapy or liver transplantation for nonresectable liver metastases from colorectal cancer? Annals of surgery. 2015;261(5):956-60.

5. Line PD, Hagness M, Berstad AE, Foss A, Dueland S. A Novel Concept for Partial Liver Transplantation in Nonresectable Colorectal Liver Metastases: The RAPID Concept. Ann Surg. 2015;262(1):e5-9.

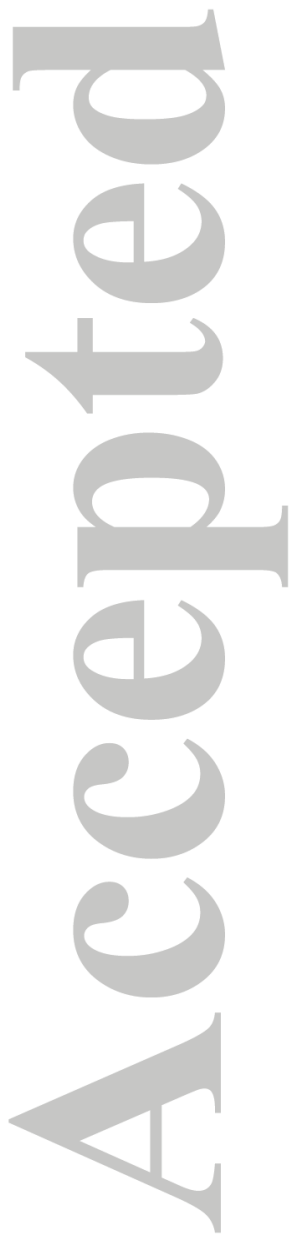

John Wiley \& Sons, Inc.

This article is protected by copyright. All rights reserved. 


\section{Table 1: characteristics of patients}
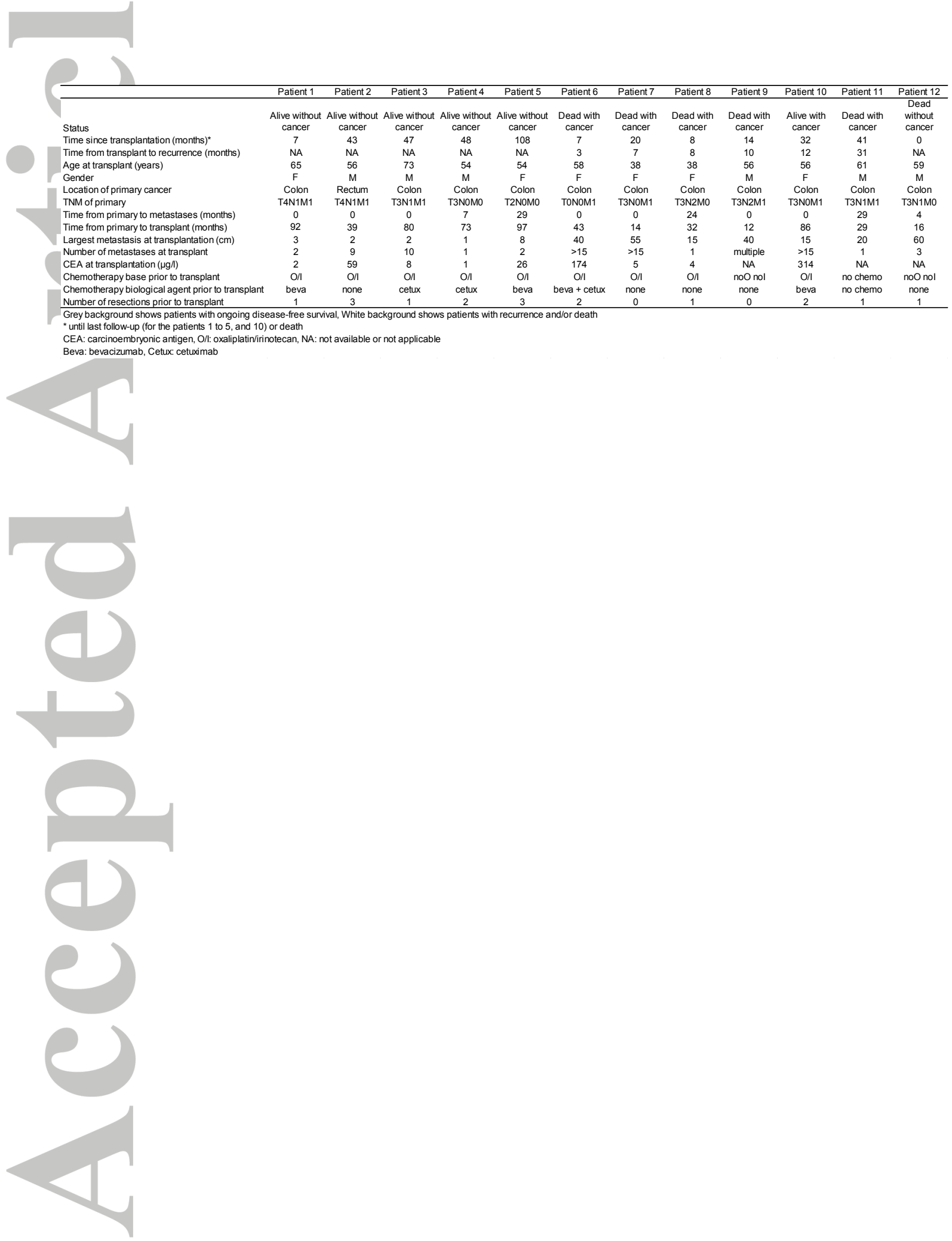

John Wiley \& Sons, Inc.

This article is protected by copyright. All rights reserved. 
Figure legends

Figure 1: Overall (full line) and disease-free (dashed line) survivals
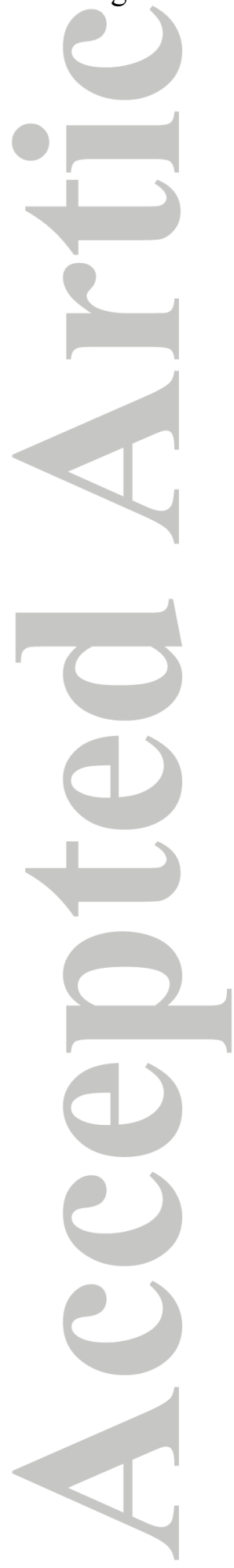

John Wiley \& Sons, Inc.

This article is protected by copyright. All rights reserved. 


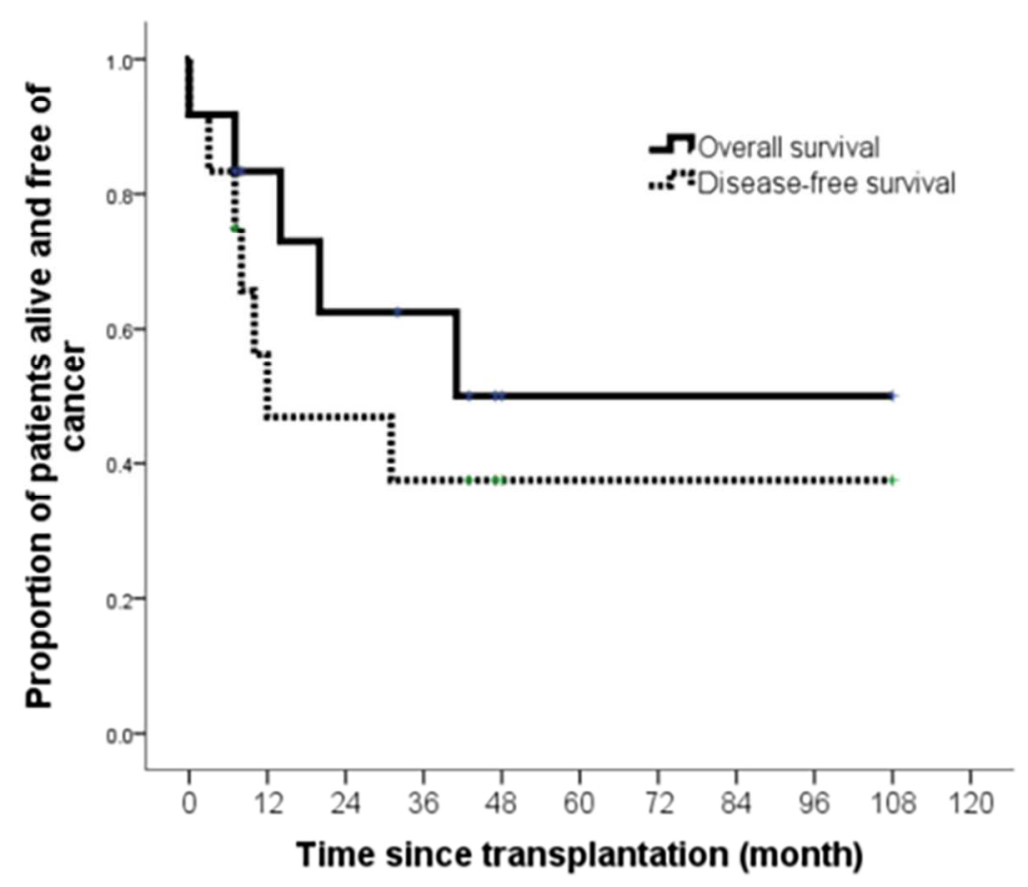

$\begin{array}{lllllll}\text { Overall survival } & 12 & 8 & 6 & 5 & 2 & 1\end{array}$

$\begin{array}{lllllll}\text { DFS } & 12 & 6 & 5 & 4 & 2 & 1\end{array}$

$114 \times 92 \mathrm{~mm}(300 \times 300$ DPI $)$

John Wiley \& Sons, Inc.

This article is protected by copyright. All rights reserved. 